\title{
Settling Time Design and Parameter Tuning Methods for Finite-Time P-PI Control
}

\author{
Keigo Hiruma ${ }^{1}$, Hisakazu Nakamura ${ }^{1}$ and Yasuyuki Satoh ${ }^{2}$ \\ 1. Department of Electrical Engineering, Tokyo University of Science, Noda 278-8510, Japan \\ 2. Graduate school of Informatics, Kyoto University, Kyoto 606-8501, Japan
}

\begin{abstract}
High precision position control and high speed control of the robot manipulators are fundamental and important control problems. The effectiveness of finite-time P-PI control was confirmed by end-effector position control of robot manipulators. However, parameter tuning method has not been proposed to finite-time P-PI control. In this paper, we propose a settling time design method and a parameter tuning method for the finite-time P-PI control. The effectiveness of the proposed parameter tuning method is confirmed by experiments of end-effector position control of a robot manipulator.
\end{abstract}

Key words: Nonlinear control, finite-time control, P-PI control, settling time design, robot manipulator.

\section{Introduction}

High precision position control and high speed control of robot manipulators are fundamental and important control problems. P-PI cascade control is commonly used for robot manipulator control [1].

In recent years, nonlinear finite-time control [2] that guarantees convergence to a desired state within finite-time attracts much attention in nonlinear control theory [3-9]. In particular, superior control performance of finite-time P-PI control was confirmed by end-effector position control of robot manipulators [10]. However, a parameter tuning method has not been developed to the finite-time P-PI control.

In this paper, we propose a settling time design method for the finite-time P-PI control based on Refs. [9] and [11]. Then, we confirm the effectiveness of the proposed method for settling time design by computer simulation.

Moreover we extend our proposed method to parameter tuning. The effectiveness of the proposed parameter tuning method is confirmed by experiments of end-effector position control of a robot manipulator.

Corresponding author: Keigo Hiruma, research fields: nonlinear control and robotics.

\section{Preliminaries}

In this section, we summarize definitions and fundamental properties of nonlinear finite-time control. Throughout the paper, $\mathbb{R}_{>0}=[0,+\infty),|| x||=$ $\left(\sum_{i=1}^{n}|x|^{2}\right)^{1 / 2}$ for all $x \in \mathbb{R}, \mathrm{B}_{\delta}=\{x \mid\|x\|<\delta\}$ and $\bar{B}_{\delta}=\{x \mid\|x\| \leq \delta\}$.

\subsection{Stability and Convergence Rate [10]}

In this subsection, we show definitions of stability and convergence rates. We consider the following differential equation:

$$
\dot{x}=f(x),
$$

Where $x \in \mathbb{R}^{n}, f: \mathbb{R}^{n} \rightarrow \mathbb{R}^{n}$ is continuous, and $f(0)=0$. Stability of the origin of Eq. (1) and convergence rate are defined as follows:

Definition 1. (Stability) The origin of Eq. (1) is said to be:

- stable for each $\varepsilon>0$ there exists $\delta>0$ such that

$$
\left\|x_{0}\right\|<\delta \Rightarrow\|x(t)\|<\varepsilon ; \forall t \geq 0 .
$$

- globally asymptotically stable if the origin is stable and all solution $x(t)$ satisfy the following equation:

$$
\lim _{t \rightarrow \infty}\|x(t)\|=0
$$


Definition 2. (Convergence rate) The origin of Eq. (1) is said to be:

- rationally stable if the origin is stable and there exists positive constant $\delta, b_{1}, b_{2}>0$ and $0<\eta<1$ such that

$$
\begin{gathered}
\|x(t)\| \leq b_{1}\left(1+\left\|x_{0}\right\|^{b_{2}} t\right)^{\frac{1}{b_{2}}}\left\|x_{0}\right\|^{\eta}, \\
\forall t \geq 0, \forall x_{0} \in B_{\delta}
\end{gathered}
$$

- exponentially stable if the origin is stable and there exists positive constant $\delta, \mathrm{b}_{1}, \mathrm{~b}_{2}>0$ such that

$$
\|x(t)\| \leq b_{1} e^{-b_{2} t}\left\|x_{0}\right\|, \forall t \geq 0, \forall x_{0} \in B_{\delta} ;
$$

- finite-time stable if the origin is stable and there exists a positive constant $\delta>0$ and a function $\mathrm{T}: \mathrm{B}_{\delta} \backslash\{0\} \rightarrow \mathbb{R}_{>0}$ such that

$$
\lim _{t \rightarrow T\left(x_{0}\right)} x(t)=0, \forall x_{0} \in B_{\delta} .
$$

\subsection{Nakamura’s Settling Time Design Method [9]}

In this subsection we consider the following chain integrator system:

$$
\dot{x}(t)=A_{c} x(t)+B_{c} u(t),
$$

where $x \in \mathbb{R}^{n}$ is a state, $u \in \mathbb{R}$ is an input, matrices $A_{c} \in \mathbb{R}^{n \times n}$ and $B_{c} \in \mathbb{R}^{n \times 1}$ are defined as follows:

$$
A_{c}=\left[\begin{array}{ccccc}
0 & 1 & 0 & \cdots & 0 \\
0 & 0 & 1 & \cdots & 0 \\
\vdots & \vdots & \vdots & \ddots & \vdots \\
0 & 0 & 0 & \cdots & 1 \\
0 & 0 & 0 & \cdots & 0
\end{array}\right], B_{c}=\left[\begin{array}{c}
0 \\
0 \\
\vdots \\
0 \\
1
\end{array}\right]
$$

For system (7), Nakamura et al. [9] proposed the following nonlinear settling time design method:

Proposition 1: (Proposition 1 in Ref. [9])

Consider system (7) and static nonlinear feedback control $u=q(x)$ such that the origin of the closed loop system is finite-time stable and $T_{\max }$ defined by the following equation is given for some $\delta>0$.

$$
T_{\max }=\max _{x_{0} \in \bar{B}_{\delta}} T\left(x_{0}\right) .
$$

Then, the following input finite-time stabilizes the origin of Eq. (7) and guarantees settling time $T_{d}$ for all $x_{0} \in \bar{B}_{\delta}$ :

$$
u=\frac{q(S x)}{d_{1}^{n} d_{21}}
$$

where $d_{1}, d_{2}$ and $S$ are defined as follows.

$$
d_{1}=\frac{T_{d}}{T_{\max }},
$$

$$
d_{2}=\left\{\begin{array}{cc}
1 & \left(d_{1} \leq 1\right) \\
d_{1}^{1-n} & \left(d_{1}>1\right)
\end{array}\right.
$$

$$
S=\operatorname{diag}\left(d_{21}, d_{1} d_{21}, \cdots, d_{1}^{n-1} d_{21}\right)
$$

\subsection{Finite-Time P-PI Control [10]}

In this subsection, we introduce finite-time P-PI control. We consider the following linear control system:

$$
\dot{x}_{1}=x_{2},
$$

$$
\dot{x}_{2}=b u+\theta
$$

where $x=\left[x_{1}, x_{2}\right]^{T} \in \mathbb{R}^{2}$ is a state, $u \in \mathbb{R}$ is an input, $b$ is a known and $\theta$ is an unknown constants.

Theorem 1: Consider the following finite-time P-PI controller for system (14)-(15):

$$
\begin{gathered}
u=-k_{2}\left|u_{0}\right|^{\frac{2}{3}} \operatorname{sgn}\left(u_{0}\right)-k_{3} \int_{0}^{t}\left|u_{0}\right|^{\frac{1}{3}} \operatorname{sgn}\left(u_{0}\right) d \tau-\frac{\hat{\theta}}{b}, \\
u_{0}=x_{2}+k_{1}\left|x_{1}\right|^{\frac{3}{4}} \operatorname{sgn}\left(x_{1}\right),
\end{gathered}
$$

where $u_{0} \in \mathbb{R}$ denotes velocity error, $k_{1}, k_{2}$ and $k_{3}$ are positive constants as design parameters, $\hat{\theta}$ is estimated value of $\theta$.

If the origin of the close-loop system is asymptotically stable, the origin is finite-time stable.

Remark 1: Note that controller (16)-(17) is different from Eq. (17) in Ref. [10] with respect to the existence of $\hat{\theta}$. However, the proof of Proposition 1 is still valid, and Theorem 1 holds. 


\section{Settling Time Design for Finite-time P-PI Control}

In this section, we propose a finite-time P-PI controller that guarantees the desired settling time.

We propose a settling time design method for the finite-time P-PI controller for linear control system (14)-(15) and then we propose a parameter tuning method based on the proposed settling time design method for the finite-time P-PI controller.

\subsection{Problem Statement}

In this subsection, we show the problem statement to be discussed in this paper.

Consider system (14)-(15) and the finite-time P-PI controller (16)-(17). Suppose that the settling time $T\left(x_{0}\right)$ for a certain initial state $x_{0}$ is known with given parameters $k_{1}, k_{2}, k_{3}$ and $\hat{\theta}$.

The objective of this paper is to design a controller to achieve the desired settling time $T_{d}$ for the initial state $x_{0}$ under the above hypotheses.

For this objective we refine the finite-time P-PI controller (16)-(17) based on Proposition 1.

Note that the approach is heavily dependent on the initial state $x_{0}$. This implies that we can design the settling time of the finite-time controlled system in the limited manner.

\subsection{Finite-Time P-PI Control of Linear Control System}

In this subsection, we propose a finite-time P-PI controller that guarantees the desired settling time for linear control system (14)-(15) in the following main theorem of the paper.

Theorem 2: Consider system (14)-(15) and finite-time P-PI controller (16)-(17).

Assume that parameters $k_{1}, k_{2}$ and $k_{3}$ asymptotically stabilize the origin, and the initial state $x_{0}$ and $\hat{\theta}$ can be written as $x_{0}=\left(x_{01}, 0\right)$ and $\hat{\theta}=\theta$, respectively. Moreover the settling time $T\left(x_{0}\right)$ is assumed to be known.

Then the following controller (18)-(19) finite-time stabilizes the origin with the settling time $T\left(x_{0}\right)=T_{d}$.

$$
\begin{gathered}
u\left(x_{1}, x_{2}\right)=-k_{2}\left(\frac{T\left(x_{0}\right)}{T_{d}}\right)^{2}\left|\bar{u}_{0}\right|^{\frac{2}{3}} \operatorname{sgn}\left(\bar{u}_{0}\right) \\
-\left.k_{3}\left(\frac{T\left(x_{0}\right)}{T_{d}}\right)^{3} \int_{0}^{t} \bar{u}_{0}\right|^{\frac{1}{3}} \operatorname{sgn}\left(\bar{u}_{0}\right) d \tau-\frac{\hat{\theta}}{b} \\
\bar{u}_{0}=\frac{T_{d}}{T\left(x_{0}\right)} x_{2}+k_{1}\left|x_{1}\right|^{\frac{3}{4}} \operatorname{sgn}\left(x_{1}\right) .
\end{gathered}
$$

To prove Theorem 2 we prepare the following three lemmas.

Lemma 1: Assume that for controller (18)-(19) the settling time for the initial state $x_{0}$ is $T\left(x_{0}\right)$.

Consider the following coordinates transformation $\phi: x \mapsto \bar{x}$ :

$$
\begin{gathered}
\bar{x}_{1}=x_{1} \\
\bar{x}_{2}=\frac{T_{d}}{T} x_{2} \\
\bar{x}_{3}=-b k_{3} \frac{T}{T_{d}} \int_{0}^{t}\left|u_{0}\left(\bar{x}_{1}, \bar{x}_{2}\right)\right| \operatorname{sgn}\left(u_{0}\left(\bar{x}_{1}, \bar{x}_{2}\right)\right) d t+\theta-\hat{\theta}
\end{gathered}
$$

and new time scale $\bar{t}=\left(T\left(x_{0}\right) / T_{d}\right) t$, where $T_{d} \in \mathbb{R}$ is a constant and $t$ is actual time.

Then for an initial state $x_{0}$ on $\bar{x}$-coordinates the controller (18)-(19) possesses $x\left(T_{d}\right)=0$.

Proof. We consider system (14)-(15) and the finite-time P-PI controller (16)-(17).

The close-loop system is obtained as follows:

$$
\begin{gathered}
\dot{x}_{1}=x_{2}, \\
\dot{x}_{2}=-b k_{2}\left|u_{0}\right|^{\frac{2}{3}} \operatorname{sgn}\left(u_{0}\right)+x_{3}, \\
\dot{x}_{3}=-b k_{3}\left|u_{0}\right|^{\frac{1}{3}} \operatorname{sgn}\left(u_{0}\right),
\end{gathered}
$$

where $x_{3}$ is defined as follows:

$$
x_{3}=-b k_{3} \int_{0}^{t}\left|u_{0}\right|^{\frac{1}{3}} \operatorname{sgn}\left(u_{0}\right) d \tau+\theta-\hat{\theta} .
$$

Let a solution of the differential Eqs. (23)-(25) starting at $x$ be $\varphi(t ; x)$.

By using coordinates transformation (20)-(22) and our proposed finite-time controller (18)-(19), we 
obtain the close-loop system as follows:

$$
\begin{gathered}
\frac{d \bar{x}_{1}}{d \bar{t}}=\bar{x}_{2}, \\
\frac{d \bar{x}_{2}}{d \bar{t}}=-b k_{2}\left|\bar{u}_{0}\right|^{\frac{2}{3}} \operatorname{sgn}\left(\bar{u}_{0}\right)+\bar{x}_{3}, \\
\frac{d \bar{x}_{3}}{d \bar{t}}=-b k_{3}\left|\bar{u}_{0}\right|^{\frac{1}{3}} \operatorname{sgn}\left(\bar{u}_{0}\right)
\end{gathered}
$$

Recall that $\varphi(t ; x)$ is a solution for Eqs. (23)-(25), and the solution of the differential Eqs. (27)-(29) is $\varphi(\bar{t} ; \bar{x})$.

Since $\varphi\left(t=T ; x=x_{0}\right)=0$ in Eqs. (23)-(25), $\varphi\left(\bar{t}=T ; \bar{x}=x_{0}\right)=0$ is in Eqs. (27)-(29).

This completes the proof.

Lemma 2. Suppose that assumptions in Lemma 1 are satisfied. Assume that for system (14)-(15) controller (18)-(19) finite-time stabilizes the origin.

Then controller (18)-(19) finite-time stabilizes the origin in both time scale $t$ and $\bar{t}$.

Proof: The close-loop system with controller (18)-(19) is the same as one with controller (16)-(17).

Note that coordinates transformation $\varphi$ is diffeomorphic, and Lemma 2 holds.

Lemma 3. Suppose that assumptions in Lemmas 1 and 2 are satisfied.

Then the initial state $x_{0}$ on $\bar{x}$-coordinates the settling time is $T_{d}$ in time scale $t$ by controller (18)-(19).

Proof. By using the time scale transformation $\bar{t}=\left(T_{d} / T\right) t$,

$$
\bar{t}=T=\frac{T}{T_{d}} t \Leftrightarrow t=T_{d}
$$

The controller (18)-(19) settles the state at the origin at $t=T_{d}$.

Proof of Theorem 2. For the initial state $x_{0}=\left(x_{01}, 0\right)$ and $\hat{\theta}=\theta$, an initial state of the close-loop system (27)-(29) is denoted as $x_{0}=\left(x_{01}, 0\right)$.

This implies that in this case the initial state is transformed into the same state by coordinates transformation $\varphi$.
According to Lemma 1, the settling time of the close-loop system (27)-(29) is $T\left(x_{0}\right)$ in time scale $\bar{t}$.

According to Lemma 3, the settling time of the close-loop system (27)-(29) is $T\left(x_{0}\right)=T_{d}$ in time scale $t$.

By Lemma 2, controller (18)-(19) finite-time stabilizes the origin.

This completes the proof.

By the same discussion as the proof of Proposition 1 in Ref. [9] (Proposition 1 in this paper) the following corollary of Theorem 2 holds.

Corollary 1. Consider system (14)-(15) and the finite-time P-PI controller (16)-(17). Suppose that $T_{\max }$ defined by the following equation is given for some $\delta>0$ and $k_{1}, k_{2}$ and $k_{3}$ :

$$
T_{\max }=\max _{\left(x_{0}, \theta\right) \in \bar{B}_{\delta}} T\left(x_{0}, \theta\right) .
$$

Then, the following input finite-time stabilizes the origin of (14)-(15) and guarantees settling time $T_{d}$ for each initial state $x_{0}=\left(x_{01}, x_{02}, \theta\right) \in \bar{B}_{\delta}$ :

$$
\begin{gathered}
u=-k_{2}\left(\frac{T_{\max }}{T_{d}}\right)^{2}\left|\bar{u}_{0}\right|^{\frac{2}{3}} \operatorname{sgn}\left(\bar{u}_{0}\right) \\
-\left.k_{3}\left(\frac{T_{\max }}{T_{d}}\right)^{3} \int_{0}^{t} \bar{u}_{0}\right|^{\frac{1}{3}} \operatorname{sgn}\left(\bar{u}_{0}\right) d \tau-\frac{\hat{\theta}}{b}, \\
\bar{u}_{0}\left(x_{1}, x_{2}\right)=\frac{T_{d}}{T_{\max }} x_{2}+k_{1}\left|x_{1}\right|^{\frac{3}{4}} \operatorname{sgn}\left(x_{1}\right) .
\end{gathered}
$$

\subsection{Parameter Tuning Method}

In this subsection, we propose a parameter tuning method by using the proposed settling time design method for finite-time P-PI control.

We replace the controller (18)-(19) of $T_{d} / T=k_{0}$.

Then, we obtain the following a new finite-time controller (34)-(35) for system (14)-(15).

$$
\begin{gathered}
u=-\frac{k_{2}}{k_{0}{ }^{2}}\left|\bar{u}_{0}\right|^{\frac{2}{3}} \operatorname{sgn}\left(\bar{u}_{0}\right) \\
-\frac{k_{3}}{k_{0}{ }^{3}} \int_{0}^{t}\left|\bar{u}_{0}\right|^{\frac{1}{3}} \operatorname{sgn}\left(\bar{u}_{0}\right) d \tau-\frac{\hat{\theta}}{b},
\end{gathered}
$$




$$
\bar{u}_{0}=k_{0} x_{2}+k_{1}\left|x_{1}\right|^{\frac{3}{4}} \operatorname{sgn}\left(x_{1}\right),
$$

where $k_{0}>0, k_{1}, k_{2}, k_{3}$ and $\hat{\theta}$ are design parameters.

Corollary 2. Assume that for system (14)-(15) controller (18)-(19) finite-time stabilizes the origin with given parameters $k_{1}, k_{2}, k_{3}$ and $\hat{\theta}$.

Then for each $k_{0}>0$ controller (34)-(35) finite-time stabilizes the origin for the initial state $x_{0}$.

Note that if $k_{0}<1$ the settling time becomes smaller and if $k_{0}>1$ one becomes larger.

\section{Computer Simulation}

In this paper, we proposed the controller that guarantees the desired settling time in Theorem 2. To ensure the basic idea of the proposed method, we consider a settling-time design problem for a simple second-order linear system.

We consider the following linear control system.

$$
\begin{gathered}
\dot{x}_{1}=x_{2}, \\
\dot{x}_{2}=u+1.0 .
\end{gathered}
$$

For system (36)-(37), we consider the following nonlinear finite-time controller:

$$
\begin{gathered}
u=-5.0\left|u_{0}\right|^{\frac{2}{3}} \operatorname{sgn}\left(u_{0}\right)-5.0 \int_{0}^{t}\left|u_{0}\right|^{\frac{1}{3}} \operatorname{sgn}\left(u_{0}\right) d \tau-\hat{\theta}, \\
u_{0}=x_{2}+2.0\left|x_{1}\right|^{\frac{3}{4}} \operatorname{sgn}\left(x_{1}\right) \\
\hat{\theta}=1.0 .
\end{gathered}
$$

Fig. 1 illustrates time histories of the state for the initial state $x_{0}=\left[x_{01}, x_{02}\right]=[-5.0,0]$. By Fig. 1, we can find that the settling time $T=3.338 \mathrm{~s}$. We set the desired settling time $T_{d}=1.50 \mathrm{~s}$ for the initial state $x_{0}=\left[x_{01}, x_{02}\right]=[-5.0,0]$. We can obtain the following input:

$$
\begin{gathered}
u=-24.760\left|u_{0}\right|^{\frac{2}{3}} \operatorname{sgn}\left(u_{0}\right) \\
-55.100 \int_{0}^{t}\left|u_{0}\right|^{\frac{1}{3}} \operatorname{sgn}\left(u_{0}\right) d \tau-\hat{\theta},
\end{gathered}
$$

$$
u_{0}=0.449 x_{2}+2.0\left|x_{1}\right|^{\frac{3}{4}} \operatorname{sgn}\left(x_{1}\right)
$$

$$
\hat{\theta}=1.0
$$

Fig. 2 illustrates respective time histories of the state with the input (41)-(43).

Though the input by Eqs. (41)-(43) is much bigger than the original one, we can permit that the state is settled at the origin at $T=1.50 \mathrm{~s}$.

We confirmed that our proposed method can achieve the desired settling time.

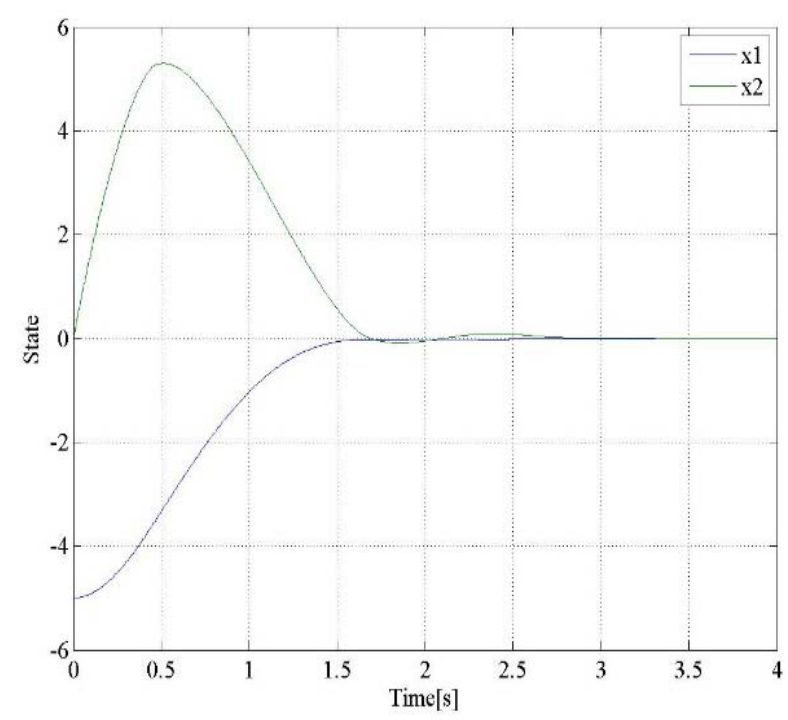

Fig. 1 Original controller: State.

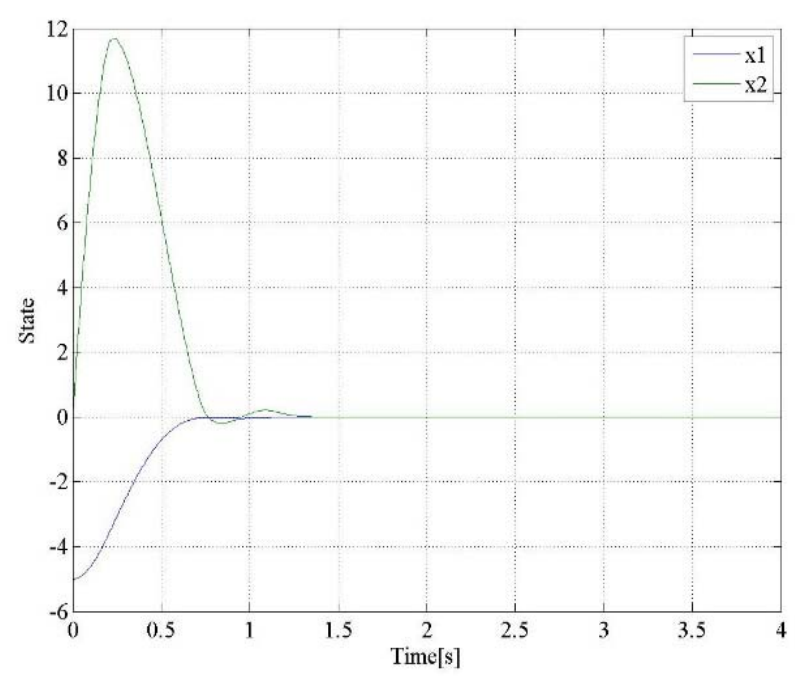

Fig. 2 Modified controller guaranteeing $T_{d}=1.5[s]$ : State. 


\section{Application to Robot Manipulator}

In this section, we apply our proposed method to a position control of a robot manipulator.

\section{1 -link Robot Manipulator System [10]}

In this subsection we consider a serial $n$-link robot manipulator modeled by the following equation:

$$
M(\phi) \ddot{\phi}+C(\phi, \dot{\phi}) \dot{\phi}+G(\phi)+F(\dot{\phi})=\tau
$$

where $\phi=\left[\phi_{1}, \cdots, \phi_{n}\right]^{T} \in \mathbb{R}^{n}$ consists of $n$-joint angles, $M$ represents the inertia matrix, $C: \mathbb{R}^{n} \times$ $\mathbb{R}^{n} \rightarrow \mathbb{R}^{n \times n}$ denotes the centripetal-Coriolis matrix, $G: \mathbb{R}^{x} \rightarrow \mathbb{R}^{n} \quad$ represents the gravity effects, $F: \mathbb{R} \rightarrow \mathbb{R}^{n}$ denotes the friction effects, and $\tau=\left[\tau_{1}, \cdots, \tau_{n}\right]^{T} \in \mathbb{R}^{n}$ is a torque input vector. This paper considers nonredundant robot manipulator; $n \leq 6$ is supposed and $q=\left[q_{1}, \cdots, q_{n}\right]^{T} \in \mathbb{R}^{n} \quad$ denotes the end-effector position in a task-space. $q$ and $\phi$ have the following relation:

$$
q=h(\phi)
$$

where $h: \mathbb{R}^{n} \rightarrow \mathbb{R}^{n}$ denotes a forward kinematics. In this paper, we consider a control problem of end-effector position $q$ to constant desired position $q_{d}=\left[q_{d 1}, \cdots, q_{d n}\right]^{T}$. In this paper, we assume that the matrix

$$
J(q)=\frac{\partial h}{\partial q}(\phi),
$$

is non-singular for all $q$ in the domain of interest.

\subsection{Finite-Time P-PI Control for n-link Robot}

\section{Manipulator}

In this subsection, we extend our proposed method to end-effector position control of the $n$-link robot manipulator.

We propose the following inverse Jacobian based on nonlinear controller for position control of the robot manipulator system (44) [10].

$$
\tau=\left[\begin{array}{c}
-\frac{k_{21}}{k_{0}^{2}}\left|\bar{u}_{01}\right|^{\frac{2}{3}} \operatorname{sgn}\left(\bar{u}_{01}\right) \\
-\frac{k_{31}}{k_{o}^{3}} \int_{0}^{t}\left|\bar{u}_{01}\right|^{\frac{1}{3}} \operatorname{sgn}\left(\bar{u}_{01}\right) d \tau-\hat{\theta}_{1} \\
\vdots \\
-\frac{k_{2 n}\left|\bar{u}_{0 n}\right|^{\frac{2}{3}} \operatorname{sgn}\left(\bar{u}_{0 n}\right)}{k_{0}^{2}} \\
-\frac{k_{3 n}}{k_{o}^{3}} \int_{0}^{t}\left|\bar{u}_{0 n}\right|^{\frac{1}{3}} \operatorname{sgn}\left(\bar{u}_{0 n}\right) d \tau-\hat{\theta}_{n}
\end{array}\right],
$$

\section{Experiments}

In this section we show experimental environments and results of end-effector position control by using the proposed method for a 3-DOF robot manipulator.

\subsection{Experimental Facility}

We implement the proposed controller on a robot manipulator PA-10 produced by Mitsubishi Heavy Industries, Ltd (MHI). We use three joints of PA-10 as illustrated in Fig. 3. The PA-10 equips an absolute resolver with $0.9 \times 10^{-5}$ rad resolution on each joint. The PA-10 is controlled by a PC with $2 \mathrm{~ms}$ sampling interval and limited the maximum torque.

The forward kinematics $q=[x, y, z,]^{T}=h(\phi)$ is obtained as follows:

$$
\begin{gathered}
x=l_{2} \cos \phi_{1} \sin \phi_{2}+\left(l_{1}+i_{3}\right) \cos \phi_{1} \sin \left(\phi_{2}+\phi_{3}\right), \\
y=l_{2} \sin \phi_{1} \sin \phi_{2}+\left(l_{1}+l_{3}\right) \sin \phi_{1} \sin \left(\phi_{2}+\phi_{3}\right), \\
z=l_{1}+l_{2} \cos \phi_{2}+\left(l_{2}+l_{3}\right) \cos \left(\phi_{2}+\phi_{3}\right),
\end{gathered}
$$




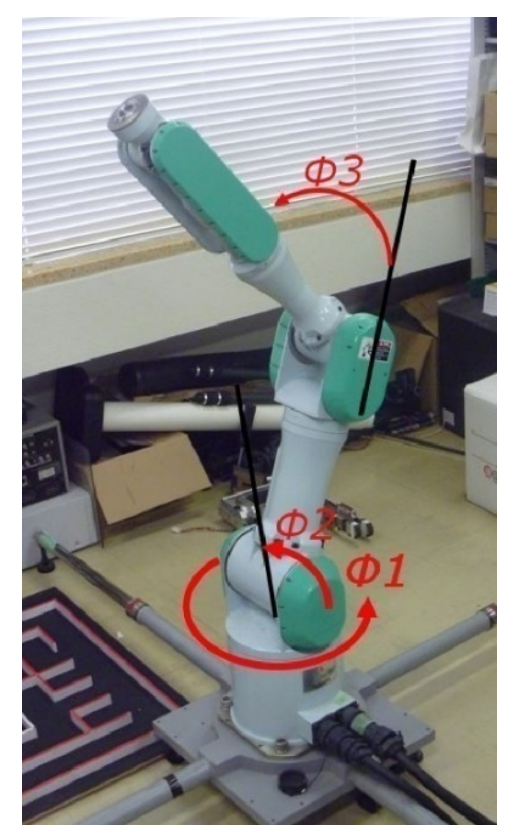

Fig. 3 Robot Manipulator PA-10.

where $l_{1}=0.317 \mathrm{~m}, l_{2}=0.45 \mathrm{~m}$ and $l_{3}=0.55 \mathrm{~m}$ are link lengths of PA-10.

In experiments, the control objective is asymptotic stabilization of the end effector position $q$ at the desired position $\left[x_{d}, y_{d}, z_{d}\right]^{T}=[0.2,-0.3,0.6]^{T}[\mathrm{~m}]$. In the experiments, we choose $\left[k_{11}, k_{12}, k_{13}\right]=[15,15,15],\left[k_{21}, k_{22}, k_{23}\right]=[450,550,350]$ and $\left[k_{31}, k_{32}, k_{33}\right]=[150,220,180]$. These parameters are used in experiments of Nakamura et al. [11].

\subsection{Experiment of Proposed Finite-Time P-PI Control}

In this subsection, we show experimental results of end-effector position control of the robot manipulator by using our parameter tuning method to the finite-time P-PI controller.

In the case of $k_{0}=0.975$, Figs. $4-6$ illustrate time histories of inputs, state variables and position control error respectively.

Tables 1 and 2 summarize the performances of the controllers with variations of $k_{0}$ from $45 \mathrm{~s}$ to $55 \mathrm{~s}$.

According to Tables 1 and 2, our parameter tuning method can perform $0.27 \mu \mathrm{m}$ in mean error and 1.0 $\mu \mathrm{m}$ in standard deviation.

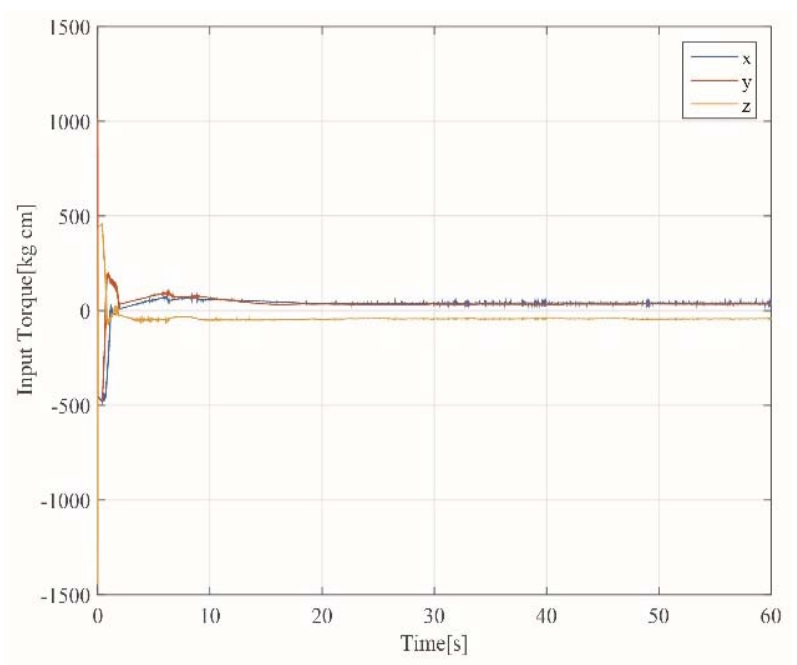

Fig. 4 Experimental result of proposed FT-P-PI: Input.

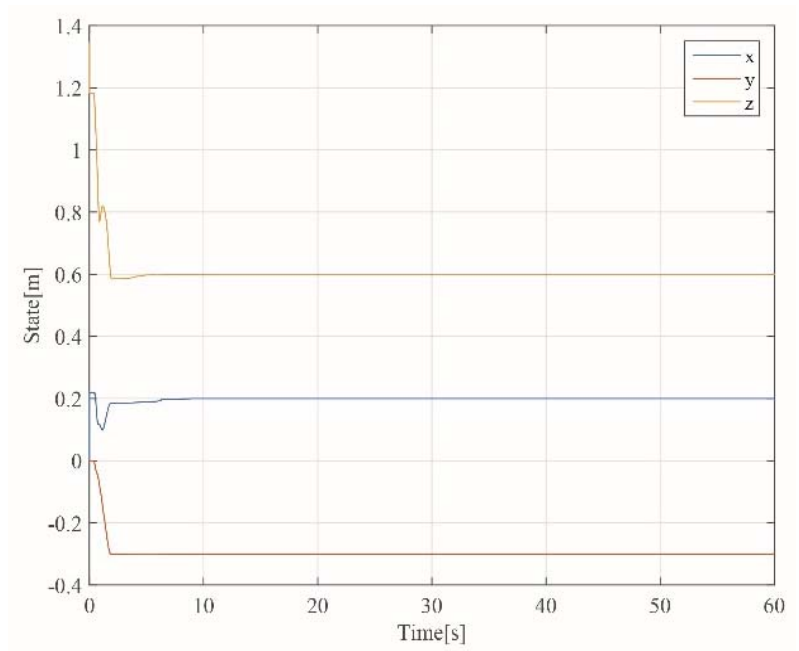

Fig. 5 Experimental result of proposed FT-P-PI: State.

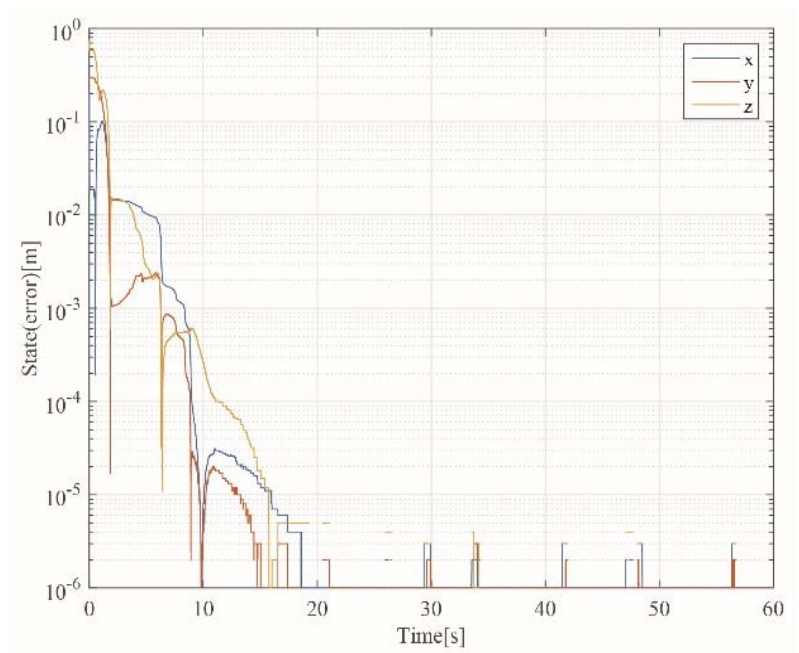

Fig. 6 Experimental result of proposed FT-P-PI: State (Error). 
Table 1 Results of main experiments (standard deviation).

\begin{tabular}{lllll}
\hline$k_{0}$ & 0.95 & 0.9625 & 0.975 & 1.00 \\
\hline$x[m]$ & $1.079 \mathrm{e}-6$ & $1.101 \mathrm{e}-6$ & $1.027 \mathrm{e}-6$ & $1.227 \mathrm{e}-6$ \\
$y[m]$ & $6.324 \mathrm{e}-7$ & $6.514 \mathrm{e}-7$ & $6.514 \mathrm{e}-7$ & $8.8408 \mathrm{e}-7$ \\
$z[m]$ & $1.130 \mathrm{e}-6$ & $9.109 \mathrm{e}-7$ & $9.109 \mathrm{e}-7$ & $2.990 \mathrm{e}-6$ \\
\hline
\end{tabular}

Table 2 Results of main experiments (mean error).

\begin{tabular}{lllll}
\hline$k_{0}$ & 0.95 & 0.9625 & 0.975 & 1.00 \\
\hline$x[m]$ & $8.193 \mathrm{e}-6$ & $3.336 \mathrm{e}-7$ & $6.624 \mathrm{e}-8$ & $1.855 \mathrm{e}-7$ \\
$y[m]$ & $2.575 \mathrm{e}-7$ & $2.261 \mathrm{e}-7$ & $2.756 \mathrm{e}-7$ & $2.342 \mathrm{e}-7$ \\
$z[m]$ & $1.126 \mathrm{e}-7$ & $3.639 \mathrm{e}-7$ & $4.013 \mathrm{e}-8$ & $7.703 \mathrm{e}-7$ \\
\hline
\end{tabular}

6.3 Experiment of Conventional Finite-Time P-PI Control

In this subsection, we show experimental results of end-effector position control of the robot manipulator by conventional finite-time P-PI control.

Figs. 7, 8 and 9 illustrate time histories of inputs, state variables and position control error respectively.

Table 3 summarizes the performances with respect to mean error and standard deviation from $45 \mathrm{~s}$ to $55 \mathrm{~s}$.

According to Table 3, conventional method can perform $0.77 \mu \mathrm{m}$ in mean error and $3.0 \mu \mathrm{m}$ in standard deviation.

\subsection{Experiment of Conventional P-PI Control}

In this subsection, we show experimental results of end-effector position control of the robot manipulator by conventional P-PI control.

In the conventional method, since it is not possible to use the parameters of the finite-time control, we choose

$$
\begin{gathered}
{\left[k_{11}, k_{12}, k_{13}\right]=[10,10,10],} \\
{\left[k_{21}, k_{22}, k_{23}\right]=[900,900,500],}
\end{gathered}
$$

and $\left[k_{31}, k_{32}, k_{33}\right]=[400,350,200]$.

Figs. 10-12 illustrate time histories of inputs, state variables and position control error respectively.

Table 4 summarizes the performances with respect to mean error and standard deviation from $45 \mathrm{~s}$ to $55 \mathrm{~s}$.

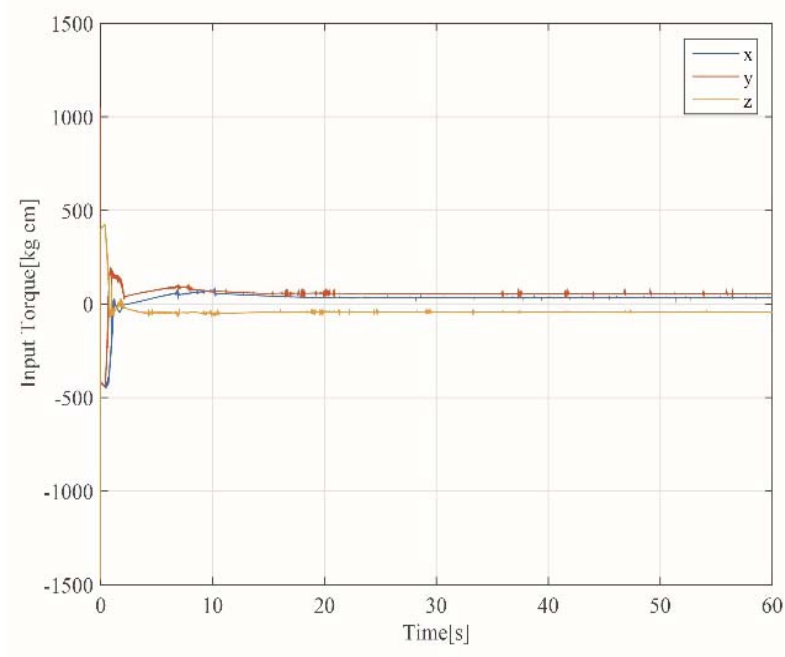

Fig. 7 Experimental result of conventional FT-P-PI: Input.

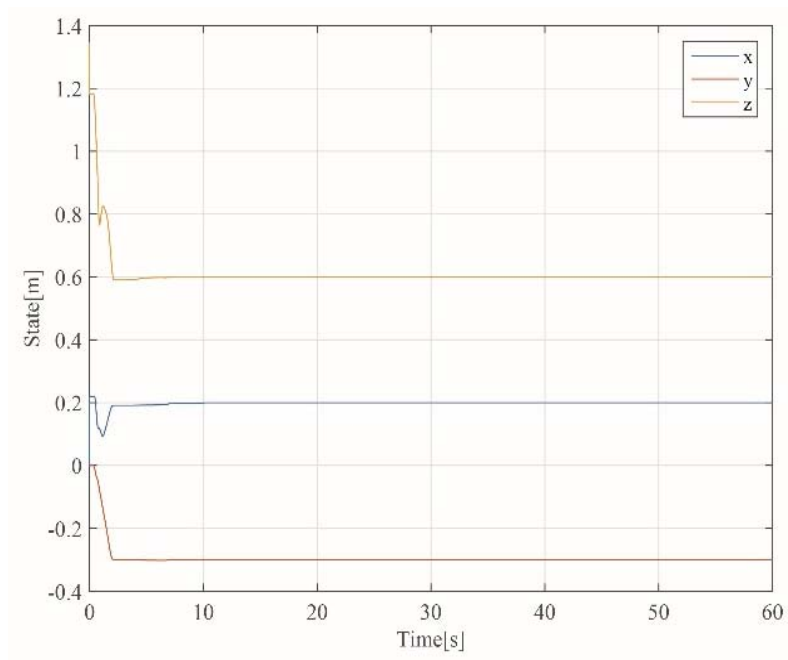

Fig. 8 Experimental result of conventional FT-P-PI: State.

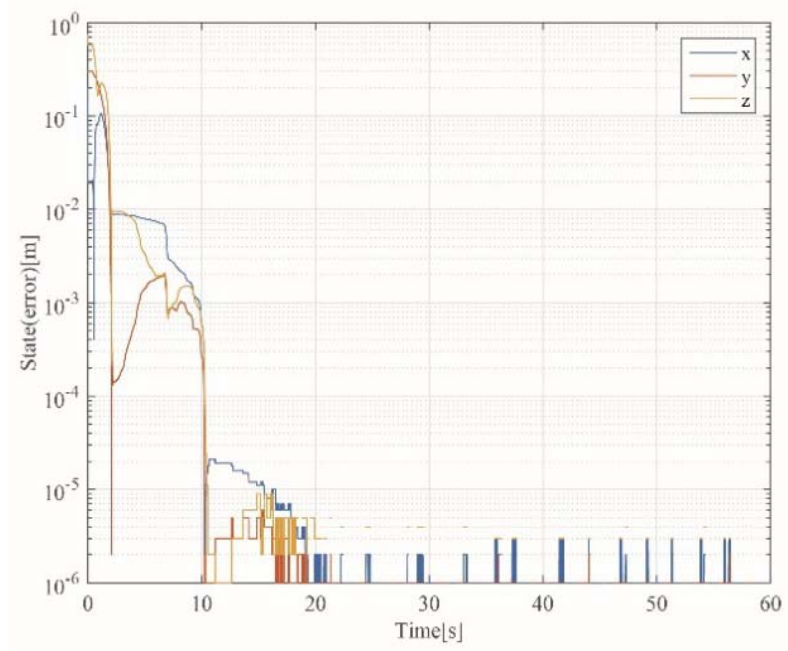

Fig. 9 Experimental result of conventional FT-P-PI: State (error). 
Table 3 Results of conventional finite-time P-PI experiments.

\begin{tabular}{llll}
\hline$k_{0}$ & $x[\mathrm{~m}]$ & $y[\mathrm{~m}]$ & $z[\mathrm{~m}]$ \\
\hline $\begin{array}{l}\text { Mean error } \\
\begin{array}{l}\text { Standard } \\
\text { deviation }\end{array}\end{array}$ & $1.855 \mathrm{e}-7$ & $2.342 \mathrm{e}-7$ & $7.703 \mathrm{e}-7$ \\
\hline
\end{tabular}

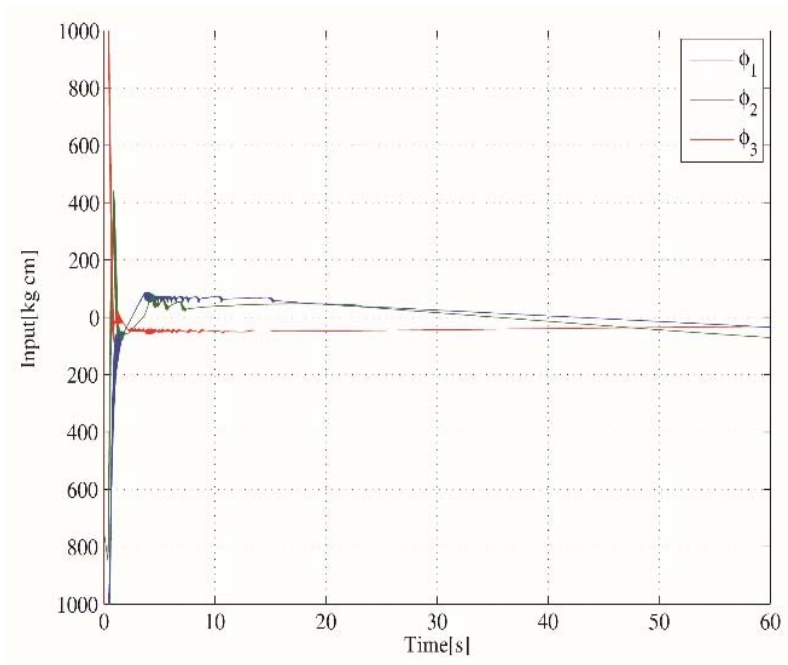

Fig. 10 Experimental result of conventional P-PI: Input.

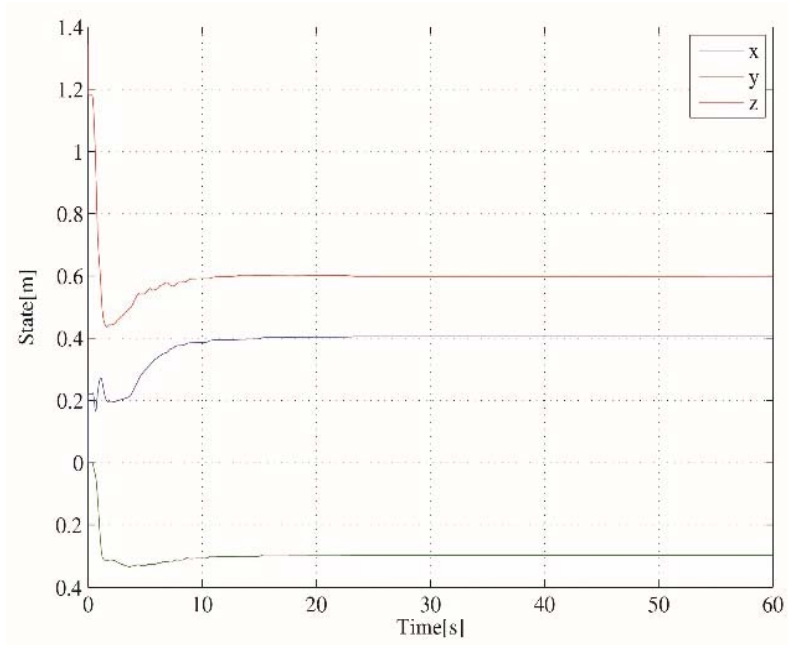

Fig. 11 Experimental result of conventional P-PI: State.

According to Table 4, the conventional P-PI control can perform $4.6 \mu \mathrm{m}$ in mean error and $5.8 \mu \mathrm{m}$ in standard deviation.

\subsection{Discussion}

Fig. 13 illustrates the standard deviations. We can confirm that the standard deviation is improved by changing $k_{0}$. This confirms effectiveness of our proposed parameter tuning method. In particular, we

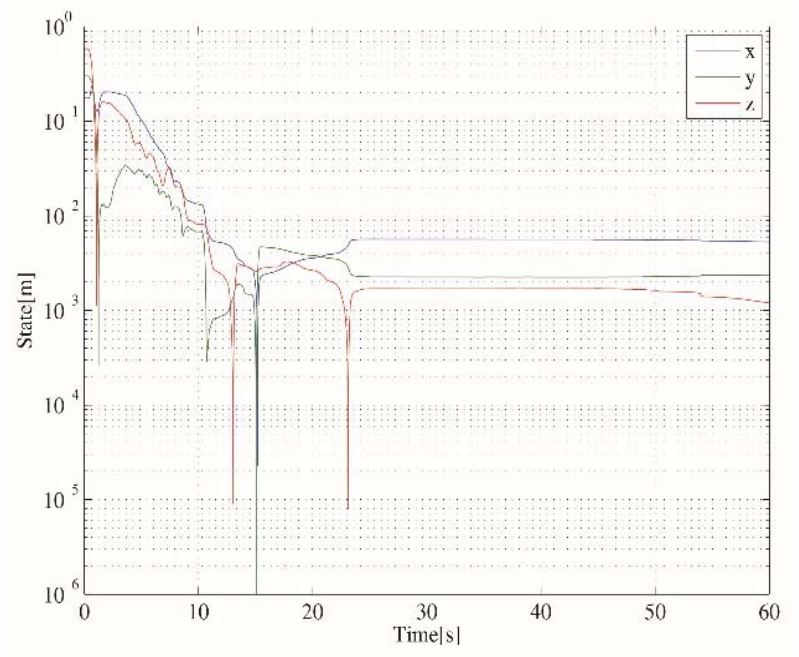

Fig. 12 Experimental result of conventional P-PI: State (Error).

Table 4 Results of conventional P-PI experiments.

\begin{tabular}{llll}
\hline$k_{0}$ & $x[\mathrm{~m}]$ & $y[\mathrm{~m}]$ & $z[\mathrm{~m}]$ \\
\hline $\begin{array}{l}\text { Mean error } \\
\begin{array}{l}\text { Standard } \\
\text { deviation }\end{array}\end{array}$ & $4.609 \mathrm{e}-6$ & $1.951 \mathrm{e}-6$ & $1.689 \mathrm{e}-6$ \\
\hline
\end{tabular}

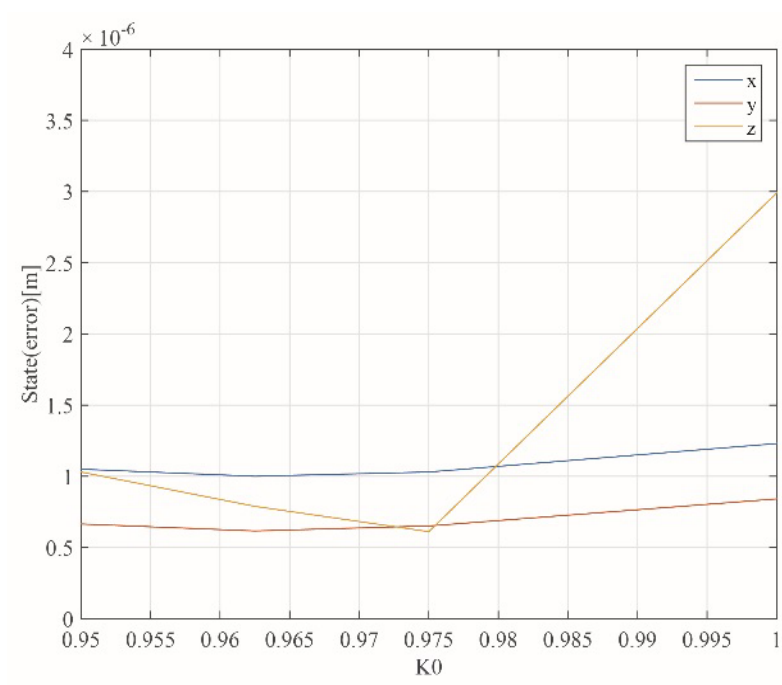

Fig. 13 Accuracy of state error in each $k_{0}$.

can see that the accuracy of the $\mathrm{x}$-axis is greatly improved when we choose $k_{0}=0.975$.

Table 5 illustrates the result of settling times. Because $k_{0} \mathrm{~s}$ are close to 1 in all cases, the settling times are not changed so much. We can see that the settling times of the finite-time control are smaller than the conventional method, in all cases. On the contrary, mean error has been greatly improved. 
Table 5 Results of settling time $( \pm 2 \%)$.

\begin{tabular}{llll}
\hline$k_{0}$ & $x$ axis[s] & $y$ axis[s] & $z$ axis[s] \\
\hline $\begin{array}{l}\text { Conventional } \\
\begin{array}{l}\text { P-PI } \\
\text { Finite-Time P-PI }\end{array}\end{array}$ & 6.4677 & 10.3119 & 8.5951 \\
$k_{0}=1.0$ & 1.9753 & 2.0285 \\
$\begin{array}{l}\text { Finite-Time P-PI } \\
k_{0}=0.975\end{array}$ & 6.5648 & 1.9543 & 2.5822 \\
$\begin{array}{l}\text { Finite-Time P-PI } \\
k_{0}=0.9625\end{array}$ & 6.3740 & 1.8151 & 3.6399 \\
$\begin{array}{l}\text { Finite-Time P-PI } \\
k_{0}=0.95\end{array}$ & 5.9891 & 1.7453 & 3.7523 \\
\hline
\end{tabular}

\section{Conclusions}

In this paper, we proposed a settling time design method for finite-time P-PI control. The proposed method can design the desired settling time and the effectiveness is confirmed by computer simulation.

For end-effector position control of the robot manipulator, our proposed method can improve accuracy of the end-effector position.

This paper does not discuss tracking control problem. This remains future work.

\section{Acknowledgement}

This work was supported by JSPS KAKENHI Grant Number $15 \mathrm{H} 04022$.

\section{References}

[1] Sciavicco, L., and Siciliano, B. 2000. Modeling and Control of Robot Manipulators. London:
Sprinver-Verlag.

[2] Haimo, V. T. 1986. "Finite Time Controllers." SIAM J. Control and Optimization 24 (4): 760-70.

[3] Bhat, S. P., and Bemstein, D. S. 1995. "Lyapunov Analysis of Finite-Time Differential Equations." In Proceedings of the American Control Conference, 1831-2.

[4] Bacciotti, A., and Rosier, L. 2005. Liapunov Functions and Stability in Control Theory. London: Sprinver-Verlag.

[5] Levant, A. 2005. "Homogeneity Approach to High-Order Sliding Mode Design." Automatica 41: 823-30.

[6] Nakamura, N., Nakamura, H., and Nishitani, H. 2011. "Global Inverse Optimal Control with Guaranteed Convergence Rates of Input Affine Nonlinear Systems." IEEE Transactions on Automatic Control 56 (2): 358-69.

[7] Orlov, Y. V. 2009. Discontinuous Systems. London: Springer Verlag.

[8] Polykov, A. 2012. "Nonlinear Feedback Design for Fixed-Time Stabilization of Linear Control Systems." IEEE Transactions on Automatic Control 57 (8): 2106-10.

[9] Nakamura, H., Nakamura, N., and Fuji, Y. 2012. "Settling Time Design for Nonlinear Finite-time Control Systems." In Proceedings of IEEE Conference on Decision and Control, 4574-9.

[10] Nakamura, H., Nishida, N., and Nakamura, N. 2012. "High Precision Control of Robot Manipulators via Finite-time P-PI Control." In Proceedings of IEEE Conference on Decision and Control, 550-5.

[11] Hiruma, K., Matsuo, Y., Sato, Y., and Nakamura, H. 2014. "Settling Time Design for Homogeneous Finite-time PID Control.” In Proceedings of SICE Annual Conference, 1522-6. 